\title{
Comportamento reológico e colagem de suspensões de porcelanas de ossos com feldspato e vidro reciclado
}

\author{
(Rheological behavior and casting of bone porcelain slips with \\ feldspar and waste glass)
}

\author{
L. A. Carús, L. Bento, S. R. Bragança \\ Departamento de Materiais, Universidade Federal do Rio Grande do Sul, Av. Osvaldo Aranha 99/705, Porto \\ Alegre, RS, Brasil 90035-190 \\ laucarus@gmail.com
}

\begin{abstract}
Resumo
A crescente preocupação ambiental trouxe grande interesse na reutilização e reciclagem de materiais. Dentro desse contexto, está o propósito do presente trabalho de estudar a utilização de um resíduo de embalagens de vidro em porcelana de ossos. Especificamente, foi analisado o comportamento reológico de formulações de porcelana de ossos, nas quais o fundente feldspato foi parcialmente substituído por vidro reciclado. Avaliou-se a reologia em relação ao comportamento da viscosidade e da tensão de cisalhamento em função da taxa de cisalhamento, e da viscosidade em função da concentração de dispersante, considerando-se a especificidade de cada matéria-prima. As peças coladas foram avaliadas em relação à facilidade de desmoldagem, tempo de formação de parede e qualidade visual do colado. Obteve-se uma barbotina com o vidro com estabilidade e comportamento reológico próximo da de feldspato.
\end{abstract}

Palavras-chave: porcelana de ossos, colagem, reologia, vidro reciclado.

Abstract

The growing environmental concern brought great interest in recycling of materials. This paper shows the use of waste glass in bone china, and the analysis of the rheological behavior of bone china compositions, in which the feldspar flux was partially replaced by recycled glass. We evaluated slip viscosity, shear stress, shear rate, and viscosity in function of dispersant addition, considering the specificity of each raw material. The casting pieces were analyzed for ease of demoulding, wall-forming time and visual quality of the parts. The slip with glass showed stability and rheological behavior similar to the feldspar slip.

Keywords: bone china, slip casting, rheology, recycled glass.

\section{INTRODUÇÃO}

As qualidades como alvura, translucidez e refletividade fizeram da porcelana de ossos um produto com um apelo estético único, altamente diferenciado das porcelanas de mesa tradicionais. Além disso, ela possui uma resistência mecânica superior. Devido a essas qualidades, a porcelana de ossos possui um mercado cativo mundial, no entanto, a crescente preocupação ambiental deverá aumentar ainda mais o interesse por este material. Produzida com $50 \%$ de ossos bovinos, matéria-prima reciclável, a porcelana de ossos poderia ser fabricada no Brasil. Isso decorre do fato de o país possuir o maior rebanho comercial do mundo, com aproximadamente 212,8 milhões de cabeças de gado, além de um parque tecnológico qualificado [1].

O crescente aumento na criação de animais de abate levou a um aumento no percentual de subprodutos dos abatedouros. Estes são gerados na cadeia produtiva da carne bovina e, geralmente, encontram aproveitamento como matéria-prima em outros segmentos da indústria. No entanto, os ossos bovinos normalmente são empregados em setores de baixa valorização, como na produção de ração animal. A manufatura de produtos cerâmicos utilizando ossos pode ser uma alternativa para a valorização desse material no mercado. A reciclagem dos subprodutos tem como finalidade diminuir os impactos ambientais e reduzir os gastos com gestão de resíduos [2]. Incluído nesse contexto, o presente trabalho propõem por meio da substituição parcial do fundente feldspato por vidro, proveniente de pequenas empresas (vidrarias), um aumento da fração reciclável de materiais-primas na produção de porcelana de ossos. $\mathrm{O}$ uso de vidro tem sido amplamente divulgado por diversos artigos publicados há alguns anos, os quais provam as vantagens da utilização do mesmo em cerâmicas triaxiais [35]. Principalmente, destaca-se a redução da temperatura de sinterização, mantendo-se as propriedades cerâmicas, como resistência mecânica e absorção de água. Recentemente, a utilização de vidro em porcelanas de ossos resultou igualmente numa significativa redução da temperatura de sinterização $[6,7]$. O processamento cerâmico de porcelanas ocorre por conformação plástica e por colagem de barbotinas. Esta técnica é realizada por via úmida, o que torna necessário 
o entendimento das propriedades reológicas das suspensões cerâmicas e a interação com a água, devido à solubilização de íons das matérias-primas. O mecanismo de estabilização das barbotinas é, em geral, mais complexo que para sistemas insaturados. Por conseguinte, a adição, substituição ou modificação de matérias-primas provoca mudanças nas propriedades reológicas da suspensão cerâmica. Para controlar o processo, é necessário manter os valores de viscosidade, tixotropia e densidade das suspensões dentro de limites estreitos, com o intuito de se obter uma maior homogeneização da batelada, redução de defeitos no produto acabado, melhor controle das propriedades cerâmicas e a redução dos custos envolvidos na produção [8].

O presente trabalho propõe um estudo comparativo da colagem e do comportamento reológico de duas formulações de porcelana de ossos, sendo uma de formulação tradicional, utilização feldspato como fundente, e outra com a adição de vidro.

\section{MATERIAIS E MÉTODOS}

Este estudo foi realizado com duas formulações, a fim de se comparar suas propriedades. A formulação de base, denominada $\mathrm{F}$, era constituída de $50 \%$ ossos calcinados, $25 \%$ caulim e $25 \%$ feldspato, em percentual mássico. A formulação de teste, denominada $\mathrm{V}$, era composta de $15 \%$ feldspato e $10 \%$ vidro reciclado, $50 \%$ ossos calcinados e $25 \%$ caulim. O pó de ossos utilizado é proveniente de uma empresa regional, cuja produção de artefatos gera um resíduo do corte e usinagem de ossos bovinos. Os ossos são tratados com soda cáustica na indústria, para a limpeza da fração gordurosa. Portanto, o presente trabalho objetiva também o aproveitamento de um resíduo, sem fins práticos à referida indústria. $\mathrm{O}$ pó de ossos foi calcinado em forno elétrico a $1000{ }^{\circ} \mathrm{C}$. O material calcinado foi moído em moinho de bolas horizontal por via úmida durante $24 \mathrm{~h}$. Posteriormente, foi levado a estufa para a secagem durante $24 \mathrm{~h}$. O vidro é um resíduo proveniente de vidrarias, recebido na forma de cacos, gerado pelo processo de corte de placas. Tratase de vidros do tipo sodo-cálcico. O feldspato e o caulim são utilizados pelas industriais locais. A Tabela I apresenta a composição química e as fases minerais e a Tabela II a distribuição granulométrica.

A caracterização físico-química das matérias-primas foi feita por fluorescência de raios X (Shimadzu XRF-1800), difração de raios $\mathrm{X}$ (Philips X'Pert), análise granulométrica (Granulômetro a Laser, Cilas 1180). A área específica foi determinada pelo método BET (Quantachrome, Nova 1000).

Para a utilização do vidro como fundente, procurou-se que os parâmetros de área superficial e granulometria dessa matéria-prima se assemelhassem com os parâmetros do fundente feldspato (Tabela II).

As suspensões tiveram seu comportamento reológico caracterizado em função da taxa de cisalhamento, viscosidade aparente, concentração de sólidos e do percentual de dispersante em solução. Os resultados foram obtidos por viscosímetro Brookfield (LVDV-II com adaptador para pequenos volumes, spindle SC4-18). Antes do ensaio, as amostras foram agitadas durante $1 \mathrm{~min}$ a uma taxa de cisalhamento de $132 \mathrm{~s}^{-1}$; posteriormente ficaram em descanso por $30 \mathrm{~s}$. As formulações foram analisadas com uma concentração de sólidos em suspensão de $70 \%$ e o dispersante utilizado foi Disperlan, Lambra S.S. As suspensões foram homogeneizadas em moinho utilizando um percentual de $0,15 \%$ de dispersante, inferior ao necessário para atingir o ponto mínimo da curva de viscosidade, mas suficiente para otimizar a homogeneização. Inicialmente, utilizou-se $5 \mathrm{~min}$ e, posteriormente, aumentou-se para $20 \mathrm{~min}$.

Testes iniciais foram realizados a fim de se obter um melhor conhecimento do comportamento das suspensões,

Tabela I - Composição química e análise de fases das matérias-primas.

[Table I - Chemical composition and phase analysis of raw materials.]

\begin{tabular}{ccccc}
\hline$\%$ em peso & Caolinita & Osso calcinado & $\begin{array}{c}\text { Feldspato } \\
\text { Potássico }\end{array}$ & $\begin{array}{c}\text { Vidro } \\
\text { reciclado }\end{array}$ \\
\hline $\mathrm{SiO}_{2}$ & 46,96 & 0,65 & 66,2 & 73,96 \\
$\mathrm{Al}_{2} \mathrm{O}_{3}$ & 38,05 & 0,8 & 16,54 & 1,59 \\
$\mathrm{Fe}_{2} \mathrm{O}_{3}$ & 0,46 & 0,12 & 0,15 & 0,18 \\
$\mathrm{MgO}$ & - & 1,1 & - & 0,2 \\
$\mathrm{CaO}$ & 0,02 & 52,2 & 0,36 & 8,37 \\
$\mathrm{Na}_{2} \mathrm{O}$ & 0,03 & 2,1 & 0,89 & 14,14 \\
$\mathrm{~K}_{2} \mathrm{O}$ & 1,14 & 0,07 & 14,66 & 0,84 \\
$\mathrm{TiO}_{2}$ & 0,03 & - & 0,03 & - \\
$\mathrm{P}_{2} \mathrm{O}_{5}$ & 0,11 & 40,5 & 0,15 & - \\
$\mathrm{Outros}$ & 0,03 & 0,2 & 0,64 & 0,71 \\
Perda ao fogo & 13,2 & 2,3 & 0,38 & 0,01 \\
& caolinita & & Quartzo & \\
Fases principais & Quartzo & Hidroxi-apatita & Microclínio & - \\
& Muscovita & & Albita & \\
\hline
\end{tabular}


Tabela II - Distribuição granulométrica das matérias-primas e das formulações de feldspato (F) e com vidro (V) e área superficial dos fundentes.

[Table II - Particle size distribution of raw materials and formulations of feldspar (F) and glass (V) and flux surface area.]

\begin{tabular}{lcccccc}
\hline \multicolumn{1}{c}{ Diâmetro $(\mu \mathrm{m})$} & Feldspato & Vidro & caulim & Pó de osso & $\begin{array}{c}\text { Formulação } \\
\text { F }\end{array}$ & $\begin{array}{c}\text { Formulação } \\
\text { V }\end{array}$ \\
\hline $10 \%<$ & 1,88 & 1,2 & 0,93 & 0,66 & 0,56 & 0,42 \\
$50 \%<$ & 18,97 & 8,03 & 4,77 & 2,21 & 4,32 & 3,40 \\
$90 \%<$ & 56,39 & 31,77 & 15,07 & 9,96 & 22,98 & 17,26 \\
Diâmetro Médio & 24,57 & 12,63 & 6,47 & 3,81 & 8,21 & 6,31 \\
Sup. Específica $\left(\mathrm{m}^{2} / \mathrm{g}\right)$ & 3,99 & 3,82 & & & & \\
\hline
\end{tabular}

visando à caracterização e otimização das condições de dispersão, tais como densidade, quantidade de dispersante, viscosidade e pH. Utilizou-se colagem em molde de gesso. As dispersões foram desenvolvidas de modo que as viscosidades das suspensões atingissem uma faixa de 600 a $900 \mathrm{cP}$, valores de viscosidade utilizados pelas indústrias de porcelanas. A qualidade das peças coladas foi avaliada pela integridade estrutural, fácil desmoldagem e tempo de formação de parede no molde de gesso.

\section{RESULTADOS E DISCUSSÃO}

Os dados registrados na Tabela III mostram que para a mesma concentração de sólidos em suspensão, a formulação $\mathrm{V}$ difere da formulação $\mathrm{F}$ por apresentar uma menor viscosidade inicial, porém uma maior espessura de parede do colado. Assim, esses resultados iniciais mostram um comportamento mais instável da barbotina da porcelana de ossos da formulação V. Resultados investigativos para a adição de silicato de sódio (dispersante) nas suspensões contendo somente ossos mostraram, igualmente, uma barbotina com um intervalo muito curto na curva viscosidade em função do percentual de dispersante [1]. Pode-se inferir que o vidro apresenta um efeito de defloculante inorgânico, o que explicaria a maior dificuldade de estabilização da barbotina, como o caso do uso de hidróxido de sódio como defloculante, o qual necessita tempos longos à estabilização [9]. Além disso, dispersantes orgânicos são melhores para suspensões de porcelanas de ossos, já que as mesmas ficam mais estáveis [10].

A instabilidade reológica de suspensões pela adição de vidro foi verificada em outro estudo, desenvolvido por um dos autores, para cerâmicas triaxiais [11]. Neste trabalho foi constatado que o vidro libera $\mathrm{Na}^{+}$e igualmente possui um efeito defloculante em barbotinas.

Com a formulação $\mathrm{V}$ não foi possível, mesmo ajustandose o teor de dispersante, controlar a rápida formação de parede e o aparecimento de trincas nas peças antes do desmolde (Fig. 1). Diferente comportamento apresentou a formulação $\mathrm{F}$, a qual possibilitou o fácil desmolde das peças.

Em razão desses resultados, optou-se pelo aumento do tempo de moagem de toda formulação V; desde modo, reduz-se a granulometria e como consequência, tem-
Tabela III - Propriedades reológicas das suspensões de feldspato (F) e vidro (V). Preparação em 5 min de moagem. [Table III - Rheological properties of suspensions of feldspar (F) and glass (V). After 5 min of milling.]

\begin{tabular}{ccc}
\hline Formulações & Formulação $\mathrm{F}$ & Formulação V \\
\hline $\begin{array}{c}\text { Densidade da } \\
\text { suspensão }\left(\mathrm{g} / \mathrm{cm}^{3}\right)\end{array}$ & 1,796 & 1,802 \\
$\begin{array}{c}\text { Formação de parede } \\
(\mathrm{mm}) \text { em } 3 \text { min }\end{array}$ & 3,58 & 5,32 \\
$\begin{array}{c}\text { Viscosidade inicial } \\
(\mathrm{cP}) \text { em } 0,7 \mathrm{~s}^{-1}\end{array}$ & 3884,0 & 2624,0 \\
\hline
\end{tabular}

se um aumento no tempo de formação de parede [9]. A redução granulométrica produz uma maior quantidade de íons lixiviáveis e ao mesmo tempo, aumenta a área superficial das partículas, aumentando, igualmente, a área reativa com os íons em suspensão. Isto deixa a previsão do comportamento reológico bastante complexo. Por outro lado, o aumento do tempo de moagem significa também um maior tempo de mistura e homogeneização entre os materiais e, ao mesmo tempo, maior interação com a água. Este é um fator chave à estabilização de uma barbotina [12, 13]. Para uma comparação direta, os mesmos parâmetros foram determinados para a formulação F, ou seja, repetiu-

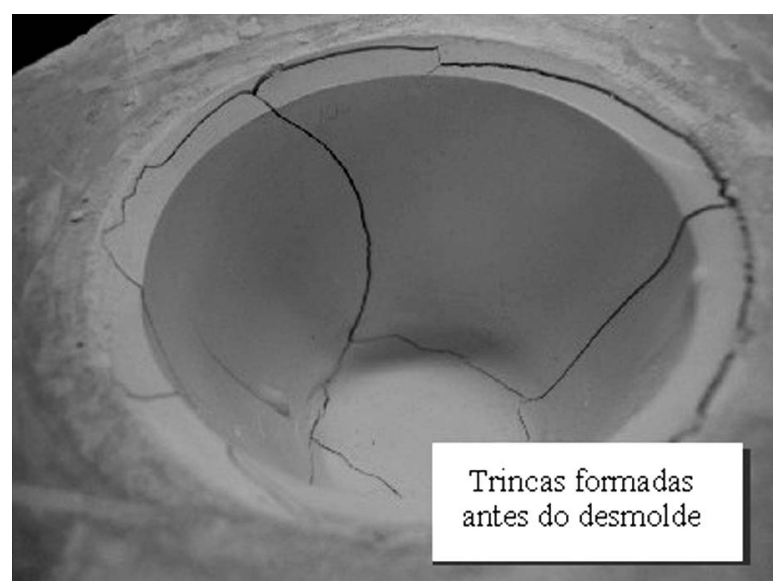

Figura 1: Trincas na peça colada da formulação de vidro (V). [Figure 1: Flaws on casted piece of glass formulation (V).] 
Tabela IV - Granulometria das formulações Feldspato (F) e Vidro (V) (20 min. de moagem).

[Table IV - Particle size of the formulations feldspar $(F)$ and glass (V) (20 min. milling).]

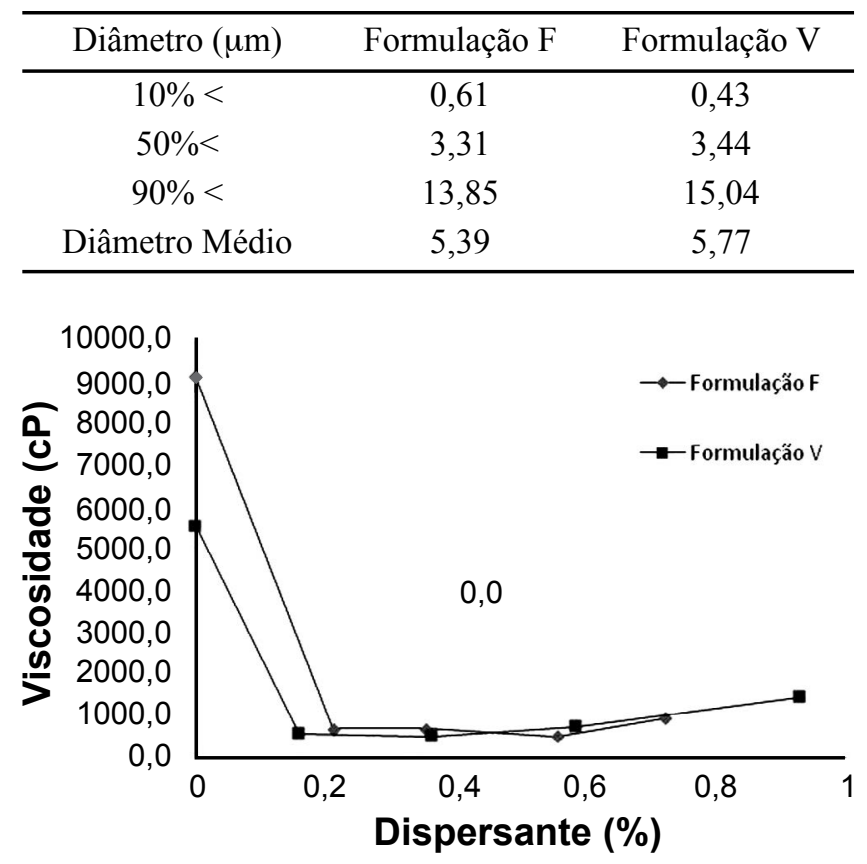

Figura 2: Viscosidade em função da quantidade de dispersante das formulações feldspato (F) e vidro (V) (taxa de cisalhamento 0,7 $\left.\mathrm{s}^{-1}\right)$. Poliacrilato de amônia.

[Figure 2: Viscosity as a function of dispersant amount of feldspar $(F)$ and glass $(V)$ Formulations (shear rate $\left.0.7 \mathrm{~s}^{-1}\right)$. Ammonia polyacrylate.]

se o mesmo tempo de mistura e moagem. Os dados de granulometria das formulações estão apresentados na Tabela IV. A Fig. 2 exibe a influência da adição de dispersante sobre a viscosidade aparente das suspensões.

$\mathrm{O}$ acréscimo de dispersante reduz a viscosidade aparente até o ponto mínimo, após, a viscosidade tende a aumentar, para as formulações. A formulação $F$ necessitou de um percentual de dispersante maior do que a formulação $\mathrm{F}$ para atingir o ponto mínimo. $\mathrm{O}$ uso de vidro favorece a defloculação da suspensão de vidro, pois esta matériaprima libera íons de hidroxila, sendo que a adsorção de $\mathrm{OH}^{-}$ sobre as partículas de caolinita promove a defloculação da barbotina, através do aumento da força de repulsão entre as partículas [14]. Foi proposta a ocorrência da lixiviação dos íons de vidro, íons alcalinos e alcalino-terrosos, levando ao aumento instantâneo no pH da suspensão [15]. Para esses autores, a dissolução do silicato só ocorre em um $\mathrm{pH}$ elevado $(\mathrm{pH}>10)$. No presente trabalho, este comportameto não é esperado, tendo em vista que o $\mathrm{pH} \cong 6$ da formulação com vidro. Testes de solubilização de pó de vidro em água, mostraram ser principalmente o sódio $(0,11 \%$ peso) que solubiliza, comparando-se com o cálcio $(0,004 \%$ peso). Este possui um fator coagulante muito superior ao do sódio. A solubilização dos íons é um processo que depende do tempo, portanto, a estabilização de uma barbotina com vidro, tornase mais difícil $[11,14]$. A variação do teor de dispersante

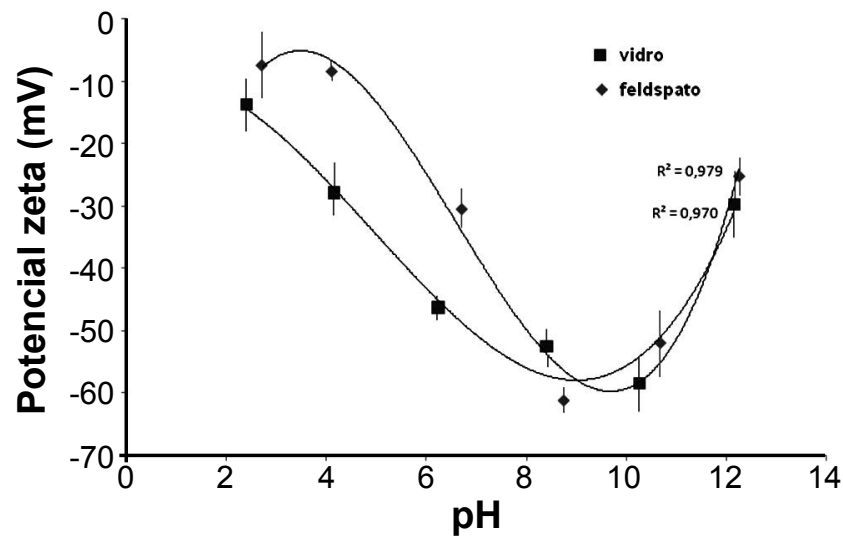

Figura 3: Curvas do potencial zeta em função do $\mathrm{pH}$, para as formulações feldspato (F) e vidro (V).

[Figure 3: Curves of the zeta potential as a function of $\mathrm{pH}$ for feldspar (F) and glass (V) formulations.]

poderia estar relacionada ao aumento no tempo de moagem da suspensão que leva ao aumento na área superficial das partículas. Entretanto, não se espera um comportamento muito diferente na moagem, entre as formulações, as quais apresentaram, praticamente, a mesma distribuição granulométrica (Tabela IV). O fato de o vidro agir como um dispersante pode ser explicado também por meio dos resultados de potencial zeta (Fig. 3).

A Fig. 3 mostra que as curvas apresentam a mesma tendência para as formulações $\mathrm{F}$ e $\mathrm{V}$. No entanto no $\mathrm{pH}$ natural das suspensões $(\sim 6)$, os valores de potencial zeta são maiores para a formulação $\mathrm{V}$. Os dados encontrados para o potencial zeta das formulações $\mathrm{F} \mathrm{e} \mathrm{V}$ foram, respectivamente, -30 e - 48. A curva de potencial zeta apresenta valores significativamente altos para um amplo intervalo de $\mathrm{pH}$, entre os valores 6 a 12 para o feldspato, e entre 4 e 12 para o vidro. Normalmente, espera-se um potencial zeta maior em módulo que -25 mv, para suspensões cerâmicas [16]. As duas suspensões atingem a mesma viscosidade aparente com um percentual de $\sim 0,3 \%$ de dispersante (Fig.

Tabela V - Propriedades das suspensões de feldspato (F) e vidro $(\mathrm{V})$.

[Table V-Proper ties of suspensions of feldspar $(F)$ and glass $(V)$.

\begin{tabular}{ccc}
\hline Propriedades & Formulação $\mathrm{F}$ & Formulação V \\
\hline Densidade $\left(\mathrm{g} / \mathrm{cm}^{3}\right)$ & 1,796 & 1,802 \\
$\begin{array}{c}\text { Área superficial do } \\
\text { Fundente }\left(\mathrm{m}^{2} / \mathrm{g}\right)\end{array}$ & 3,98 & 3,82 \\
$\begin{array}{c}\text { Espessura de parede } \\
\text { em } 3 \text { min }(\mathrm{mm})\end{array}$ & 3,14 & 3,02 \\
$\begin{array}{c}\text { Viscosidade a } 0,7 \mathrm{~s}^{-1} \\
(\mathrm{cP})\end{array}$ & 704,0 & 576,0 \\
$\begin{array}{c}\text { Tempo de escoamento } \\
(\mathrm{s})\end{array}$ & 58 & 62 \\
\hline
\end{tabular}


2). Cabe salientar que a moagem e homogeneização dessas formulações foram realizadas com $0,15 \%$ de dispersante, logo o total de dispersante necessário para se chegar ao ponto mínimo da curva foi de $0,45 \%$. Este é um valor muito bom de consumo de dispersante, tanto do ponto de vista técnico quanto econômico. Acima do ponto mínimo de viscosidade, as adições de dispersante levam ao aumento da viscosidade (Fig. 2). Este comportamento é também explicado na literatura, chamado de 'sobre-defloculação' [16].

A Tabela $\mathrm{V}$ apresenta os resultados de densidade e viscosidade aparente obtidos após a defloculação, os quais se encontram dentro dos parâmetros estimados para a produção de porcelanas. Conforme consta na referida Tabela $\mathrm{V}$, a formulação $\mathrm{V}$ apresenta uma viscosidade aparente (taxa de cisalhamento $0,7 \mathrm{~s}^{-1}$ ) menor em $\sim 130 \mathrm{cP}$ em relação a formulação $F$.

Os resultados de escoamento em copo Ford ( $\left.\mathrm{n}^{\circ} 4\right)$ e formação de parede, para as formulações $\mathrm{F} \mathrm{e} \mathrm{V}$, Tabela $\mathrm{V}$, mostram um comportamento semelhante entre as formulações. No entanto, ao se comparar os resultados de formação de parede apresentados nas Tabela III e $\mathrm{V}$ é possível observar que o tempo de moagem afetou diretamente o tempo de formação de parede. Os resultados encontrados são próximos aos utilizados pelas indústrias de porcelanas [1].

No processo de colagem a diminuição do tamanho de partículas leva a uma maior obstrução da capilaridade do molde [17]. Menores partículas levam a um menor diâmetro do raio do capilar da parede formada, o que dificulta a percolação da água [18]. Portanto, a formação de uma granulometria mais fina, em razão do maior tempo de moagem, favoreceu a redução da espessura de formação de parede. As duas formulações apresentam comportamento pseudoplástico, conforme se observa na Fig. $4 \mathrm{~A}$ viscosidade das suspensões diminui à medida que aumenta a taxa de cisalhamento e, paralelamente, aumenta a tensão de cisalhamento. Por conseguinte, a substituição

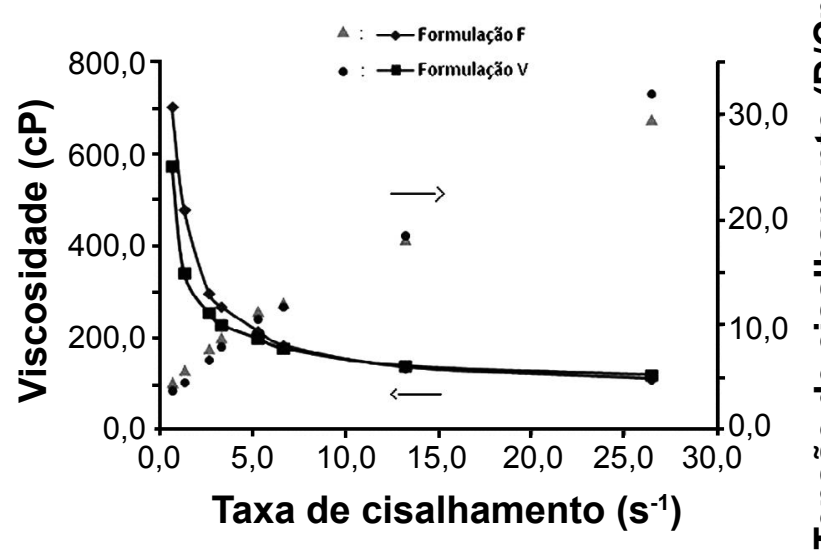

Figura 4: Curva reológica das formulações de feldspato (F) e vidro (V).

[Figure 4: Rheological curve of feldspar $(F)$ and glass (V) formulations.]

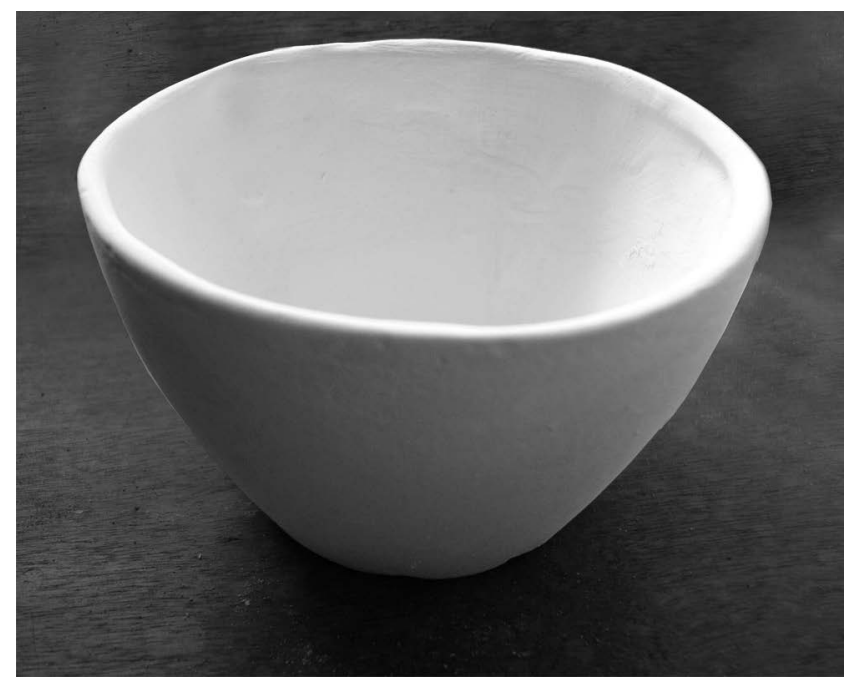

Figura 5: Peça colada da formulação de vidro (V). [Figure 5: Casted part of glass composition (V).]

parcial do feldspato pelo vidro, reduz a viscosidade inicial da barbotina, mas não altera o comportamento da mesma.

Após o aumento no tempo de moagem e homogeneização, assim como o ajuste de viscosidade aparente, a formulação $\mathrm{V}$, passou a apresentar fácil desmoldagem e boa qualidade das peças coladas (Fig. 5), fato que não ocorria anteriormente (Fig. 1).

\section{CONCLUSÕES}

$\mathrm{O}$ uso de vidro como substituto de feldspato causa um efeito defloculante em barbotinas de porcelanas de ossos e dificulta a estabilização das mesmas, prejudicando a colagem das peças. Este problema foi resolvido no presente trabalho pelo aumento do tempo de moagem e, principalmente, por um maior tempo de mistura e homogeneização entre os materiais e, ao mesmo tempo, maior interação com a água. Considerou-se este um fator chave à estabilização da referida barbotina, já que o pó de vidro apresenta íons solubilizáveis, cujo grau de solubilização é um processo dependente do tempo. O efeito de redução da viscosidade pela adição de vidro foi explicado pelo aumento do potencial zeta. Este aumento foi atribuído a formação de íon $\mathrm{OH}^{-}$os quais aumentam a força de repulsão entre partículas. A formulação com vidro atingiu valores altos de potencial zeta, de aproximadamente - $50 \mathrm{mV}$. As formulações de vidro e feldspato apresentaram comportamento reológico similar e suspensões estáveis, após a defloculação. Ambas mostram a redução da viscosidade das suspensões com o incremento da taxa de cisalhamento e, paralelamente, o aumento da tensão de cisalhamento, caracterizando curvas típicas de Bingham.

\section{AGRADECIMENTOS}

Os autores agradecem ao $\mathrm{CNPq}$ pelo apoio financeiro e bolsa de Mestrado. Ao coordenador do laboratório de materiais cerâmicos Prof. Dr. C. P. Bergmann. 


\section{REFERÊNCIAS}

[1] L. A. Carús, Diss. Mestrado, Universidade Federal do Rio Grande do Sul, RS, Brasil (2012).

[2] J. L. S. Borém, http://www.ebah.com.br, acessado em Novembro de 2012.

[3] P. R. Jackson, P. Hancock, D. Cartlidge, "Recycled bottle glass as a component of ceramic sanitaryware", M. C. Lymbachiya, J. J. Roberts, Eds., London, UK (2004).

[4] S. R. Bragança, C. P. Bergmann, J. Eur. Ceram. Soc. 24 (2004) 2383.

[5] T. Tarvornpanich, G. P. Souza, W. E. Lee, J. Am. Ceram. Soc. 88, 5 (2005) 1302.

[6] M. P. Ballvé, S. R. Bragança, Cerâmica 56 (2010) 57.

[7] L. A. Carús, S. R. Bragança, Adv. Appl. Cer. 112, 3 (2013) 169-175.

[8] C. M. da Rocha, Diss.Mestrado, Universidade de Aveiro, Portugal (2008).

[9] R. Herrmann, "Slip casting in practice", Ceramics Monographs - Handbook of Ceramics, Verlag Schimdt GmbH Freiburg i. Brg, Alemanha (1989).

[10] P. Rado, "Bone china", Ceramics Monographs - A
Handbook of Ceramics, Verlag Schmidt GmbH Freiburg i. Brg., Alemanha (1981).

[11] S. R. Bragança, C. P. Bergmann, Int. J. Appl. Ceram. Technol. 6 (2009) 264.

[12] W. E. Worral, "The Colloid Chemistry of Clays and Ceramics Materials", Ceramics Monographs - Handbook of Ceramics, Verlag Schmidt, Freiburg, Alemanha (1982).

[13] G. W. Phelps, "Slip Casting", Ceramics Monographs Handbook of Ceramics, Verlag Schmidt, Freiburg, Alemanha (1982).

[14] M. W. Carty, U. Senapati J. Am. Ceram. Soc. 81, 1 (1998) 3.

[15] W. C. La Course, W. Manson, Glaze Problems From a Glass Science Perspective, Science of Whitewares, Eds. V. E. Henkes, G. Y. Onoda, W. M. Carty, Am. Ceram. Soc., Westerville, OH, EUA (1996).

[16] J. S. Reed, "Principles of Ceramics Processing", Ed. John Wiley \& Sons., $2^{\text {nd }}$ ed. (1998).

[17] J. M. F. Ferreira, J. Eur. Ceram. Soc. 18, 9 (1998) 1161. [18] C. H. Schilling, I. A. Aksay, 'Slip Casting', ASTM Eng. Mater. Handbook, Vol. 4: Ceramics and Glasses, ASTM Int., Cleveland, OH, EUA (1991).

(Rec. 07/02/2013, Ac. 15/11/2013) 